\title{
Adjustment of Model Parameters to Estimate Distribution Transformers Remaining Lifespan
}

\author{
Victor A. Jimenez¹, Adrian Will1, Jorge Gotay¹, Sebastián Rodríguez ${ }^{1,2}$ \\ ${ }^{1}$ Grupo de Investigación en Tecnologías Informáticas Avanzadas-U.T.N.-F.R.T, Tucumán, Argentina \\ ${ }^{2}$ Consejo Nacional de Investigaciones Científicas y Técnicas, Buenos Aires, Argentina \\ Email: adrian.jimenez@gitia.org, adrian.will@gitia.org, jorge.gotay@gitia.org, sebastian.rodriguez@gitia.org
}

How to cite this paper: Jimenez, V.A., Will, A., Gotay, J. and Rodríguez, S. (2018) Adjustment of Model Parameters to Estimate Distribution Transformers Remaining Lifespan. Smart Grid and Renewable Ener$g y$, 9, 151-170.

https://doi.org/10.4236/sgre.2018.99010

Received: September 4, 2018

Accepted: September 26, 2018

Published: September 29, 2018

Copyright () 2018 by authors and Scientific Research Publishing Inc. This work is licensed under the Creative Commons Attribution International License (CC BY 4.0).

http://creativecommons.org/licenses/by/4.0/

\begin{abstract}
Currently, the electrical system in Argentina is working at its maximum capacity, decreasing the margin between the installed power and demanded consumption, and drastically reducing the service life of transformer substations due to overload (since the margin for summer peaks is small). The advent of the Smart Grids allows electricity distribution companies to apply data analysis techniques to manage resources more efficiently at different levels (avoiding damages, better contingency management, maintenance planning, etc.). The Smart Grids in Argentina progresses slowly due to the high costs involved. In this context, the estimation of the lifespan reduction of distribution transformers is a key tool to efficiently manage human and material resources, maximizing the lifetime of this equipment. Despite the current state of the smart grids, the electricity distribution companies can implement it using the available data. Thermal models provide guidelines for lifespan estimation, but the adjustment to particular conditions, brands, or material quality is done by adjusting parameters. In this work we propose a method to adjust the parameters of a thermal model using Genetic Algorithms, comparing the estimation values of top-oil temperature with measurements from 315 $\mathrm{kVA}$ distribution transformers, located in the province of Tucumán, Argentina. The results show that, despite limited data availability, the adjusted model is suitable to implement a transformer monitoring system.
\end{abstract}

\section{Keywords}

Distribution Transformer, Thermal Model, Transformer Lifespan, Parameters Adjustment, Genetic Algorithms

\section{Introduction}

The increase of energy demand caused by the consistent growth of the electricity 
consumption over the last few years caused the electricity distribution companies to begin considering the integration of the information and communication technologies with the electrical infrastructure. This situation leads to the implementation of Smart Grids, the next generation of power grids [1]. The access to real-time data from the power system state provided by the Smart Grids allows the service quality improvement and operation costs reduction. This enhancement of the service can be achieved through the application of intelligent data analysis techniques [2].

The relatively slow progress of the Smart Grids in Argentina is due to different factors, mainly the lack of proper investment by companies, and poor planning in the energy distribution context. This situation results in a progressive decrease in the service quality. The margin between installed power and the consumers demand is reduced year after year and, consequently, the electricity distribution system in Argentina is then left with a very small reserve margin, since the electricity demand approaches in many occasions the installed capacity. Particularly in the hot seasons in Argentina, summer temperatures above $35^{\circ} \mathrm{C}$ with over $90 \%$ humidity are normal, so there are corresponding consumption peaks related to the use of Air Conditioning devices massively. These peaks produce severe damage or reduction of lifespan for several distribution transformers and led to the Energetic Emergency law [3]. Against this background, the electricity distribution companies must act efficiently to avoid damaging their equipment.

In this sense, estimating and monitoring the lifespan reduction of distribution transformers is a key for decision making, since it enables companies to take the necessary actions for transformer management on time. Several actions can be taken in order to handle the situation (replacing it with a higher capacity transformer, distributing the electricity consumption to near transformers, expanding the electrical system by installing a new transformer whenever possible, etc.). But efficient resource management requires planning and prevision, so an estimate of remaining lifespan and loss rate is necessary.

However, estimating the remaining lifespan of a power transformer is not a simple task for many reasons. The accumulative effects of the transformer deterioration are not well known, so predict this expected lifespan accurately is a big challenge, even under strictly controlled conditions. Recent works performing transformer state diagnosis based on the analysis of cooling oil [4] [5], vibrations [6] and others (for a review, see [7], and [8]). Many of these publications propose an accurate diagnosis of transformer state analyzing its cooling oil, but they cannot be used in countries such as Argentina, where the Smart Grids have just arrived, because their implementation requires a high cost. A good alternative to estimate the remaining lifespan is based on several tests and experts knowledge. For this purpose, empirical life expectancy curves have been developed, founded on functional tests performed in controlled conditions, measuring the decrease of mechanical and electrical resistance under different operating conditions on commercially produced distribution transformers. These empirical results led to 
the development of general recommendations for estimating the lifespan, established in loading guides and standards, based mainly on the aging of the winding insulation [9] [10].

Over time, different models have been proposed to estimate the remaining lifespan of distribution transformers. Each of them takes into consideration different criteria and it is based on different physical effects to explain the transformer deterioration. The Chendong model [11] is an example of models that use the polymerization degree as an indicator of the degradation condition of the insulation paper. The stress generated by thermal, mechanical and electrical effects causes the reduction of the polymerization degree, which in turn reduces the mechanical resistance of the insulation paper, leading to its breaking [12]. On the other hand, there are models which consider that the aging of the winding insulation of the transformer is due mainly to internal temperature effects during its operation. The first IEEE thermal model [13] calculates the temperature of the hot-spot in the winding using the load and ambient temperature measurements. Then, from this hot spot temperature, the lifespan of the winding is estimated by comparison with respect to a nominal expected loss, determined by the insulation construction material. Based on this standard emerged new loading guides that use improved models, such as the IEC 60076-7 [9] and the IEEE C57.91-2011 [10].

Thermal models are widely used because of their simplicity of implementation (since they require only a few easy to acquire input variables). Also, the incorporation of new sensors for measuring other variables that allow better estimations of transformer remaining lifespan is limited in general and especially in Argentina since the advent of Smart Grids is relatively recent [14] [15]. Changes in technology and media support make it difficult to reliably store and retrieve historical data, as well as the complete repair history of transformers. However, despite these models are general, there is a need to adapt them to local conditions of use. This adjustment consists of determining the values of some of its parameters using known measurements. The Parameters Estimation Problem [16] is well known and appears in many areas of science, including the estimation of parameters in thermal models for estimating the internal temperature of a transformer [17]. Galdi et al. [18] use Genetic Algorithms to estimate the parameters of the thermal models. Their method uses real hot-spot temperature measurements, which are not always measure. The authors do not analyze the possibility that there is more than one suitable solution (traditional genetic algorithm is used).

This paper presents a methodology to adapt the thermal model to local conditions, to estimate the lifespan reduction in distribution transformers, based on existing loading guides. The used model takes current consumption and ambient temperature data as the only input variables, and we use information about transformer construction characteristics in the model parameter setting step. We use a Niching Genetic Algorithm to determine the best combination of parame- 
ters values in the top-oil temperature model (temperature in the upper layer of the refrigerant oil tank). To validate the model, we were applied the methodology to $315 \mathrm{kVA}$ distribution transformers, located in the province of Tucumán, Argentina, whose history was well known (they were not maintained or repaired in its history). The proposed methodology represents an improvement over existing off-the-shelf international guidelines, which takes advantage of minimal measured data and adapts to the prevailing conditions.

The rest of this paper is organized as follows: Section 2 describes the methodology used to estimate the lifespan of distribution transformers and the procedures for parameter setting, both for the top-oil temperature model and the accumulated loss of life; Section 3 describes the tests performed and the results obtained; Section 4 presents the conclusions.

\section{Materials and Methods}

\subsection{Thermal Model Based on International Loading Guides}

Although deterioration or aging of the transformer insulation is a function of temperature, time and content of moisture, oxygen and acid, the model presented in loading guides [9] [10] is based exclusively on temperature as the control parameter. Most of the aging studies focus on the effects produced by the temperature at the hottest point of the winding (called hot-spot).

The analyzed guides (IEC 60076-7 [9] and the IEEE C57.91-2011 [10]) propose two alternatives to describe the hot spot temperature as a function of time, load and ambient temperature. On the one hand, there is a simple solution that uses an exponential equation, which is appropriate when the electric load varies according to a step function, only appropriate when the electric load jumps discretely and it can be modeled by a step function. On the other hand, there is a more accurate solution based on differential equations, which is more suitable when both the charge and the ambient temperature vary arbitrarily. In our particular situation, we decided to use the solution based on differential equations, since the models will be used for transformers monitoring, where there are continuous measurements of the variables available.

\subsubsection{Estimation of the Hot-Spot Temperature Using Differential Equations}

The models described in the loading guides analyzed are designed to explain the hot-spot temperature dynamics, in power transformers. For this purpose, they take into account two effects: the heating caused by the electrical currents flowing through the winding, and the effects of the temperature changes due to the oil flow moving around the hot spot. Then, as an intermediate step for estimating the temperature in the hot-spot, we must first determine the oil temperature on the top of the container, where the flow is at its hottest. Figure 1 shows a block diagram that describes the necessary steps for the hot-spot temperature estimation procedure, taking the load factor $K$ (dimensionless parameter) and ambient temperature $\theta_{a}\left[{ }^{\circ} \mathrm{C}\right]$ measurements as input variables. 


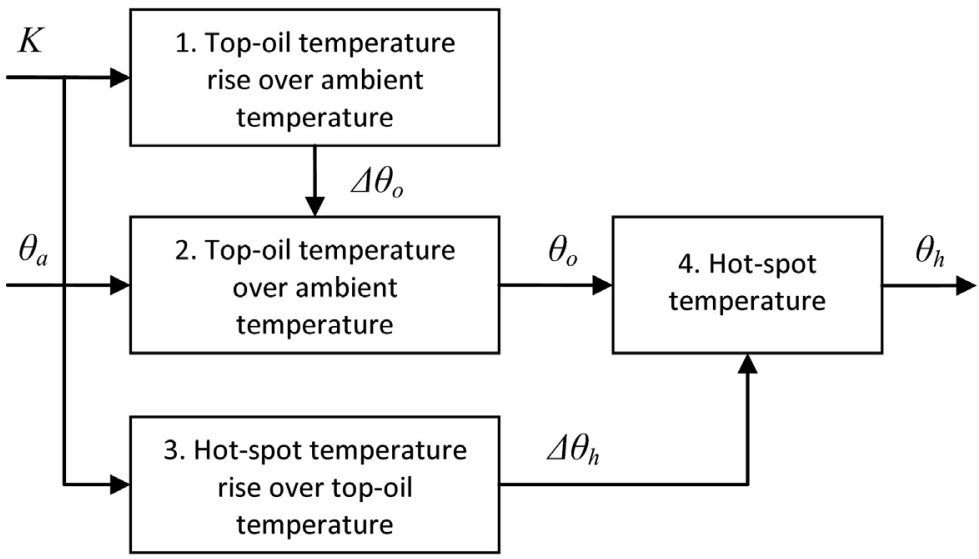

Figure 1. Block diagram of the hot-spot temperature estimation procedure used to describe the temperature of the hottest point inside the transformer $\theta_{h}$ as a function of load factor $K$ and ambient temperature $\theta_{a}$. The top-oil temperature $\theta_{o}$ and hot-spot temperature rise $\Delta \theta_{h}$ are estimated in intermediate steps.

The load factor is calculated dividing the current measurements of each phase $I_{f}[\mathrm{~A}]$ on rated current $I_{n}[\mathrm{~A}]$, which is obtained from the rated power value of three-phase distribution transformer $S[\mathrm{kVA}]$ as shows in Equation (1). For practical reasons, the nominal voltage $U_{n}=220[\mathrm{~V}]$ and the power factor $\cos (\varphi)=0.9$ are adopted, so the rated current is just obtained as $I_{n}=1.45 \times S$. Deviations on $\cos (\varphi)$ are severely fined both by governments, regulatory authorities and companies on users, son it being above 0.9 is a realistic assumption. Then, for $315 \mathrm{kVA}$ distribution transformers, the rated current $I_{n}$ is equal to 459.3 [A].

$$
I_{n}=1000 \times S /\left[2 \sqrt{3} \times U_{n} \times \cos \cos (\varphi)\right]
$$

As mentioned above, the first step in the hot-spot temperature estimation process is calculating the temperature in the cooling oil. The Equation (2) describes the dynamics of the oil temperature change on the upper level of the container (top-oil). This equation will first be used to estimate the top-oil temperature rise $\Delta \theta_{o}$, which will then be used to calculate the top-oil temperature by solving the differential equation. This procedure corresponds to step 1 in the block diagram shown in Figure 1. The inputs are the load factor $K$ and the ambient temperature $\theta_{a}\left[{ }^{\circ} \mathrm{C}\right]$, and the output is the top-oil temperature $\theta_{o} \quad\left[{ }^{\circ} \mathrm{C}\right]$.

$$
\frac{d \theta_{o}}{d t}=\frac{1}{k_{11} \times \tau_{o}}\left[\left(\frac{1+K^{2} R}{1+R}\right)^{x} \times \Delta \theta_{o r}-\left(\theta_{o}-\theta_{a}\right)\right]
$$

On the other hand, we must calculate the hot-spot temperature rise above the top-oil temperature $\Delta \theta_{h}$. To model the thermal behavior of the winding hot-spot, the loading guides propose the Equation (3) and Equation (4). The first one represents the fundamental hot-spot temperature rise, without taking into account the effect of the oil flow. The second equation represents the temperature variability that introduces the oil flow through the hot-spot, which changes 
much slower. Then, combining both effects, according to the Equation (5) we can calculate the hot-spot temperature rise. The solving of these equations corresponds to step 3 in the block diagram shown in Figure 1.

$$
\begin{gathered}
d \Delta \theta_{h 1} / d t=1 /\left(k_{22} \times \tau_{w}\right) \times\left[k_{21} \times K^{y} \times\left(\Delta \theta_{h r}\right)-\Delta \theta_{h 1}\right] \\
d \Delta \theta_{h 2} / d t=\left(k_{22} / \tau_{o}\right) \times\left[\left(k_{21}-1\right) \times K^{y} \times\left(\Delta \theta_{h r}\right)-\Delta \theta_{h 2}\right] \\
\Delta \theta_{h}=\Delta \theta_{h 1}-\Delta \theta_{h 2}
\end{gathered}
$$

Finally, the calculation of the hot-spot temperature (step 4 in the block diagram of Figure 1) is completed by the Equation (6).

$$
\theta_{h}=\theta_{o}+\Delta \theta_{h}
$$

Unfortunately, these differential equations cannot be solved analytically or exact solution in terms of simple mathematical functions. However, they can be solved using well known numerical methods. In this paper, we have selected the Runge-Kutta method using the GNU Octave Ordinary Differential Equations package [19].

\subsubsection{Relative Aging Rate}

Once the hot-spot temperature has been calculated over a time period, we can proceed to calculate the insulation degradation rate of the transformer. Figure 2 shows a box diagram that describes this procedure.

As mentioned earlier, the first step of this procedure is to estimate the hot-spot temperature, which corresponds to the dotted lines box in Figure 2, and it is described in detail in Figure 1. After that, the relative aging rate referred to the hot-spot is calculated using the Equation (7), where $B=15000$ is a valid parameter value when the transformer is constructed with thermally upgraded paper [10].

$$
V=\exp \left[B /(110+273)-B /\left(\theta_{h}+273\right)\right]
$$

Integrating the relative aging rate $V$ over a certain time period we can obtain the total lifespan $L$ occurred in that period. We use the Equation (8) when we have discrete values, where $V_{n}$ is the relative aging rate at time $n, t_{n}$ is the time duration of $n$ and $N$ is the number of intervals considered.

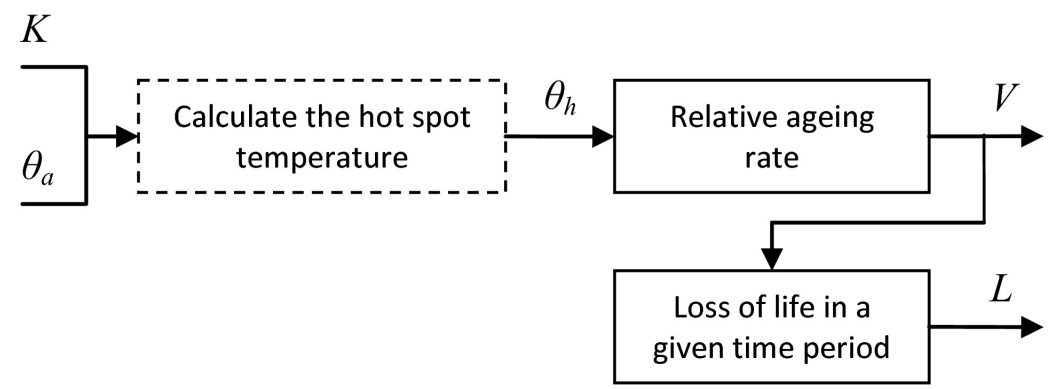

Figure 2. Block diagram of the procedure for the relative aging rate $V$ and accumulated loss of life estimation $L$, based on the load factor $K$ and ambient temperature $\theta_{a}$. The hot-spot temperature values $\theta_{h}$ are estimated in an intermediate step. 


$$
L=\int_{t_{1}}^{t_{2}} V d t \rightarrow L \approx \sum_{n=1}^{N} V_{n} \times t_{n}
$$

\subsection{Parameters for the Top-Oil Temperature Model}

The models used in the loading guides include certain parameters whose values are established based on many tests performed on the transformers under controlled conditions. Since these parameters depend on the manufacturing characteristics among other things, it is possible that the proposed values may not be suitable in all cases. For these reasons, an adjustment process of these parameters is necessary, in order to adapt the thermal model to different kinds and brands of transformers and its particular conditions. If real temperature measurements are available, the model parameters can be adjusted in such a way that the estimates obtained using the model are as close as possible to the real values. In this context, the problem is referenced as a parameter estimation problem, which belongs to the class of so-called inverse problems [16]. One of its characteristics is that they are ill-posed problems in the sense that they have more than one solution. For this reason, we decided to approach the problem using an Evolutionary Algorithm as an optimization method.

The model for the top-oil temperature estimation described in Section 2 was adjusted from measurements provided by the local electricity distribution company, Empresa de Distribución Eléctrica de Tucumán (E.D.E.T.s.a.). For this work, data were collected from two $315 \mathrm{kVA}$ distribution transformers located in residential areas with similar consumption profiles. One of these datasets was used in the parameter adjustment process, and the other one in the validation stage, where the top-oil temperature was calculated and compared with real values to determine the error obtained when using the adjusted parameters. Besides the electrical current measurement, the transformers have a temperature sensor located at the top of the oil tank. The available measurements correspond to the period between 13-11-2016 and 25-02-2017, and the records were sampled every 15 minutes. This time period corresponds to the hot season, which facilitates the model adjustment in conditions of higher ambient temperature. About the ambient temperature, the data comes from a meteorological station from the same company, located less than 1.5 kilometers away from the transformers.

\subsubsection{Statistical Analysis}

To evaluate the performance of the adjusted models, the errors are analyzed by comparing the real temperature values $\left(Y_{i}\right)$ with temperature estimations produced by the thermal model $\left(T_{i}\right)$. To do this, we use different metrics commonly used in the literature, such as: Root Mean Squared Error or RMSE (Equation (9)), whose value is interpreted easily because it is expressed in the same unit that the variable to be estimated; Percentage Root Mean Squared Error or RMSE\% (Equation (10)), which expresses the RMSE as percentage; Mean Bias Error or MBE (Equation (11)), which allow us to know if there is an underestimation or overestimation, analyzing its sign; Pearson's Correlation Coefficient $R$ 
(Equation (12)), which helps to determine the extent in which the model follows the general trend of the data.

$$
\begin{gathered}
\operatorname{RMSE}(T, Y)=\sqrt{\frac{\sum_{i=1}^{n}\left(Y_{i}-T_{i}\right)^{2}}{n}} \\
\operatorname{RMSE} \%(T, Y)=\frac{1}{Y_{\max }} \sqrt{\frac{\sum_{i=1}^{n}\left(Y_{i}-T_{i}\right)^{2}}{n}} \\
\operatorname{MBE}(T, Y)=\sum_{i=1}^{n} \frac{Y_{i}-T_{i}}{n} \\
R(T, Y)=\frac{n \sum Y_{i} T_{i}-\sum Y_{i} \sum T_{i}}{\sqrt{n \sum Y_{i}^{2}-\left(\sum Y_{i}\right)^{2}} \sqrt{n \sum T_{i}^{2}-\left(\sum T_{i}\right)^{2}}}
\end{gathered}
$$

\subsubsection{Genetic Algorithms}

Genetic Algorithm (GA) is a stochastic search technique that allows us to find solutions to a wide variety of optimization problems [20]. The GAs mimicked the genetic process of natural evolution, and they were proposed for the first time by John Holland [21]. The basic scheme of a GA begins with a population of randomly generated individuals (set of initial solutions). By applying some genetic operators, these solutions are selected, combined and mutated, so that each new population tends to be better than the previous ones, through generations. We will use AG to find the best combination of values for the parameters of the thermal models, because of their good performance in nonlinear problems involving real noisy data.

To use a GA and find solutions to the parameter adjustment problem, each individual is coded by a vector with real values, where each element represents a particular parameter that we want to find. From Equation (2) we can see that the parameters to be optimized are: $k_{11}, x, R, \Delta \theta_{o r}$ and $\tau_{o}$. However, to prevent the occurrence of different values representing the same solution we set the value of $k_{11}=1$ as indicated in Loading Guides, so that the combination $k_{11} \times \tau_{o}$ is unique. This is known as redundancy in the context of GA and heuristics, and it should be avoided since it complicates the search unnecessarily. On the other hand, to avoid inconveniences due to a possible wrong calibration or bad location of the temperature sensors (it might not be located exactly in the zone of higher oil temperature), an additional parameter is included by adding a constant $C_{\text {offset }}$ to the Equation (2). This extra parameter is used only in the solution search process in order to automatically correct a measurement offset detected in some cases. Nevertheless, we set $C_{\text {offset }}=0$ once found the best parameter values.

To delimit the search space, we define lower and upper bounds for each parameter. Then, each gene of the individuals will correspond to a single parameter to optimize, which are bounded by the limits described in Table 1. These limits were established based on the values proposed by the IEC Loading Guide [9]. Also, restricting the search space helps the algorithm to choose values that 
Table 1. Values suggested by IEC Loading Guide, Lower and Upper Bounds, and optimized values of the top-oil temperature model parameters proposed in this paper for the local conditions of use.

\begin{tabular}{ccccc}
\hline \multirow{2}{*}{ Parameter } & \multicolumn{4}{c}{ Parameter value } \\
\cline { 2 - 5 } & Lower Bound & Upper Bound & IEC Guide & Optimization \\
\hline$x$ & 0.70 & 1.10 & 0.80 & 0.94 \\
$R$ & 3.30 & 6.50 & 5.00 & 5.31 \\
$\Delta \theta_{\text {or }}$ & 40.0 & 60.0 & 55.0 & 40.6 \\
$\tau_{o}$ & 90 & 210 & 180 & 106 \\
$C_{\text {offiset }}$ & -0.20 & 0.20 & 0.00 & 0.00 \\
\hline
\end{tabular}

respect the real meaning given to each parameter of the model, that is, to make them consistent with the physical meaning they have.

\subsubsection{Niching Genetic Algorithms}

As discussed above, given the nature of the problem, the combination of parameters of a differential equation that generates a particular solution might not be unique. That is, there could be more than one combination of parameters that produce similar responses. For this reason, it is necessary to search all existing parameter sets to analyze and select the most adequate to ensure that the model generalizes adequately. In this perspective, there are GA architectures specially designed for situations where more than one solution is required. These algorithms, called Niching, allow us to find and preserve all points or zones of the search space corresponding to the local optimums. This means that the Niching algorithms will search for as many different numerical combinations as they can find, of the model parameters that allow obtaining estimates with similar error level.

Among the existing variants of Niching algorithms we use Deterministic Crowding [20] [22] because its parameter adjustment is simple, it does not require specifying the niche size and the number of generations is not a critical parameter and it is easy to obtain. The version used in this paper was implemented in Octave [19], which is described below:

- Initialization: a population is created, consisting of $n$ randomly generated individuals so that each gene will have random values within the range defined by the lower and upper limits for each of them.

- Evaluation: each individual is evaluated using the fitness function defined for the problem, described in Section 2.2.4.

- Crossover: all the individuals of the population are grouped in pairs, to recombine them and generate new individuals. We decided to apply more than one operator to achieve a good exploration of the search space as is usual in this architecture. A Uniform Crossover scheme is implemented to the half of the population so, for each gene, one child copies their value from one randomly chosen parent (the other child will take the gene value from the other 
parent). The other half of the population is generated using Cube Crossover, in which children genes are randomly generated within the range determined by the parent genes values.

- Replacement: each parent is grouped with their most similar child, and then they are compared to determine which one survives the next generation. The Euclidean distance metric is used to determine which child is the most similar to each parent, and the fitness values associated with each individual are used to decide which is best and therefore allowed to continue into the next generation (see the flow diagram in Figure 3). This mechanism reduces competition among individuals who do not belong to the same niche, thus allowing the algorithm to preserve local optima.

To set the GA parameters, some considerations were taken into account. On the one hand, the population size should be large enough to be able to adequately cover the entire search space. In this paper, this was achieved with a population of 100 individuals. On the other hand, to set the number of generations, we established as a termination condition that individuals must generate estimations that exceed a certain threshold of precision. The RMSE is used as the error metric, and the threshold is set to $5\left[{ }^{\circ} \mathrm{C}\right]$ for estimations of the top-oil temperature. Thus, the number of generations reached allows us to find solutions that generate estimations with a good accuracy, but at the same time maintain the diversity of the solutions, and do not continue after the majority of the population is made up of solutions that provide good estimations.

\subsubsection{Fitness Function}

As in nature, the selection of individuals in GA is made according to a criterion:

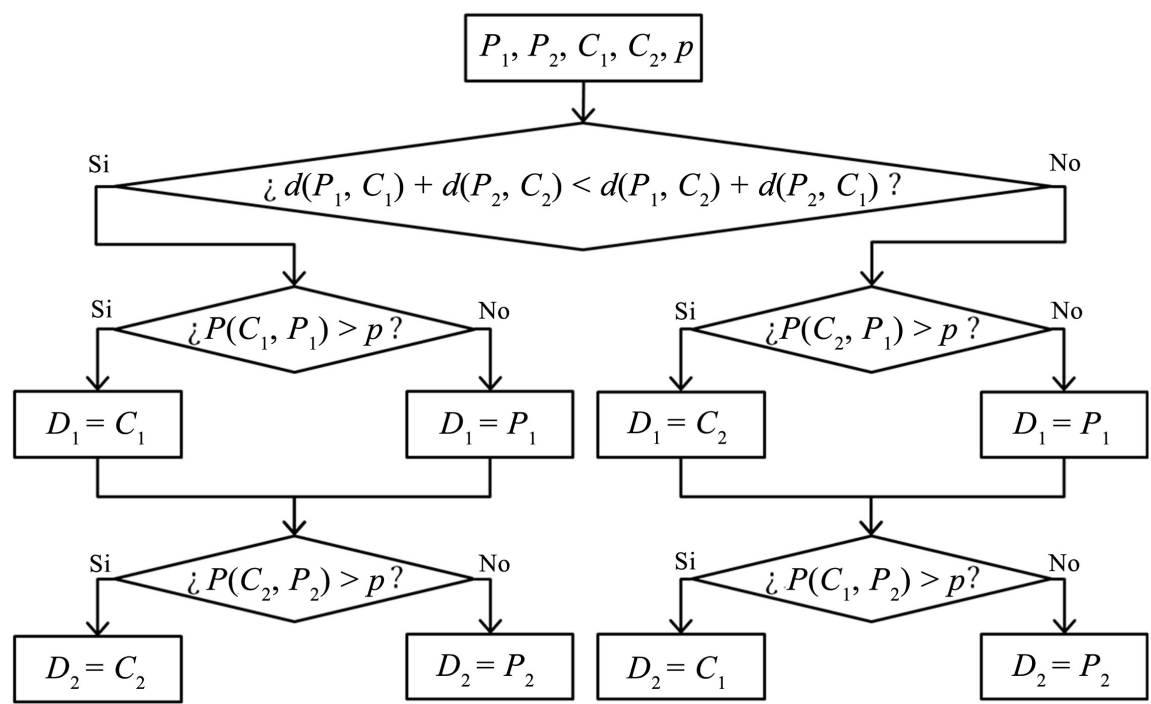

Figure 3. Replacement Step of the Deterministic Crowding algorithm. Parents $\left(P_{1}\right.$ and $\left.P_{2}\right)$, and offspring $\left(C_{1}\right.$ and $C_{2}$ ) are paired based on a distance function $d$. Then it is decided which individual in each pair survives into next generation $\left(D_{1}\right.$ and $\left.D_{2}\right)$, based on a random $p \in(0,1)$ value. 
the more suitable they are to solve the problem, the more possibilities they have to survive and reproduce. The fitness function allows us to evaluate each individual and weighs and compare them, in order to determine which solution is more adequate. Thus, the best solutions will be those that enable a better fit of the temperature estimates curve with the measured temperatures curve. Then, the fitness function (which must produce a real numeric value proportional to how good the solution is) is defined in Equation (13), where $Y(S)$ are the estimated values of the top-oil temperature using the parameters determined by the solution $S$, and $T$ represents the real measurements of top-oil temperature. Good solutions will have a low RMSE value and $R$ value close to 1 , so the fitness value will be high. The constant term in the fitness function equation is added only to ensure we do not obtain negative values.

$$
\text { fitness }(S)=50-\frac{\operatorname{RMSE}(T, Y(S))}{R(T, Y(S))}
$$

\subsection{Parameters for the Hot-Spot Temperature Model}

The same methodology used to set the parameters of the top-oil temperature equation can be used to adjust the parameters of the hot-spot temperature equations. It would require real temperature measurements of the hot spot taken over a time period to compare them with the results of the adjusted model. However, hot-spot temperature measurements are difficult to get, since the transformers should include a fiber optics sensor that is usually installed in the factory, but this is not a common situation. Furthermore, determining the exact location of the hot spot is not a simple task, since it depends on the particular characteristics of each transformer. Additionally, installing a sensor inside a transformer that does not include this factory feature generally involves dismantling it and taking it out of service for an extended time period. For these reasons, at the moment we do not have measurements of hot-spot temperature. So, we adopt the parameter values proposed by the loading guides for obtaining the first approximation of the results. Table 2 shows the values and a brief description of these parameters.

\subsection{Adjustment for the Estimation of Lifespan}

The Loading Guides propose a method for estimating the rate of aging based on the hot-spot temperature. The expected lifespan of 20 years is considered as the reference, which is the time it takes to degrade the insulation paper of the winding in normal operating conditions (100\% power consumption). From this, the Equation (7) is proposed, and the parameter $B=15,000$ is set up for the thermally upgraded paper. This equation is used to determine how fast a transformer reduces its lifespan due to the effect of its winding temperature being at its maximum or exceeded for a certain period of time. However, this reference may be different for distribution transformers with particular characteristics, depending on the type of insulation paper and operating conditions (for example if 
Table 2. Parameters to calculate the hot-spot temperature recommended by IEC Loading Guide for three-phase distribution transformer with $315 \mathrm{kVA}$ power rate and ONAN cooling system type.

\begin{tabular}{ccc}
\hline Parameter & Value & Description \\
\hline$y$ & 1.6 & Thermal exponent \\
$k_{21}$ & 1.0 & Thermal constant \\
$k_{22}$ & 2.0 & Thermal constant \\
$\tau_{w}$ & 4.0 & Winding time constant \\
$\Delta \theta_{h r}$ & 35.0 & Hot-spot to top-oil gradient at rated current \\
\hline
\end{tabular}

it operates in locations with high ambient temperature, direct solar radiation, high humidity, among others).

The hot-spot temperature measurements from the transformer installation time to the moment where it suffers some damage due to overload (i.e. over its whole lifetime) are required to find a better value for the parameter B in the Equation (7). These measurements are unusual and difficult to obtain in Argentina since the installation of new remote measuring Devices on distribution transformers is in progress, and it is relatively recent. Moreover, repair history and traceability is usually not well documented. For this reason, we propose an alternative method to do this adjustment using only the available measurements of the ambient temperature and the electric load at the transformer substation. First, the data time series are filled and completed, considering the period starting from the installation time of the transformer. The data filling procedure is performed by replicating the available data, adjusting the magnitude of the measurements according to the values of annual increments of electricity consumption recorded throughout the province [23].

After completing the data set, we estimate the hot-spot temperature and the accumulated loss of life. For the last step, it is necessary to find the value of the parameter B so that the PVU calculation is equal to $100 \%$ at the moment when we know that the transformer suffered severe damage due to overloads. For this adjustment, we have information on two $315 \mathrm{kVA}$ distribution transformers, which we know that they continuously operated until they were damaged due to overloads (without moving it or repair it). We also assume that there were no events related to failures that could accelerate the aging process (bumps, faults in the contact terminals, short-time over-voltages, among others) as a hypothesis of the problem. One of these cases is used to obtain a proper value of the parameter $\mathrm{B}$, and the other one is used to validate the results achieved with this value. The procedure described above could not be applied to more cases due to the limited information available about the distribution transformers currently installed. Only two cases can be analyzed given the current circumstances, hoping to have more cases as the remote measuring network grows and better care in keeping the history of movements and repairs. 


\section{Results and Discussion}

To use the GA described in Section 2.2.3, we used data from a particular Distribution Transformer in order to obtain a set of parameter combinations of the top-oil temperature, and then the model was applied using each combination on a different dataset, from the other transformer, to determine the most appropriate. Figure 4 shows the solutions obtained with the GA, represented in a parallel

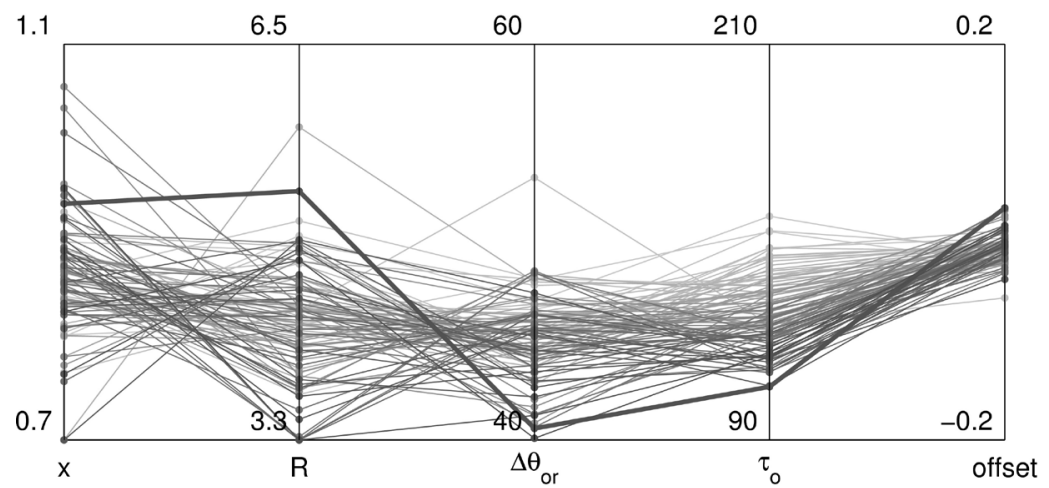

(a)
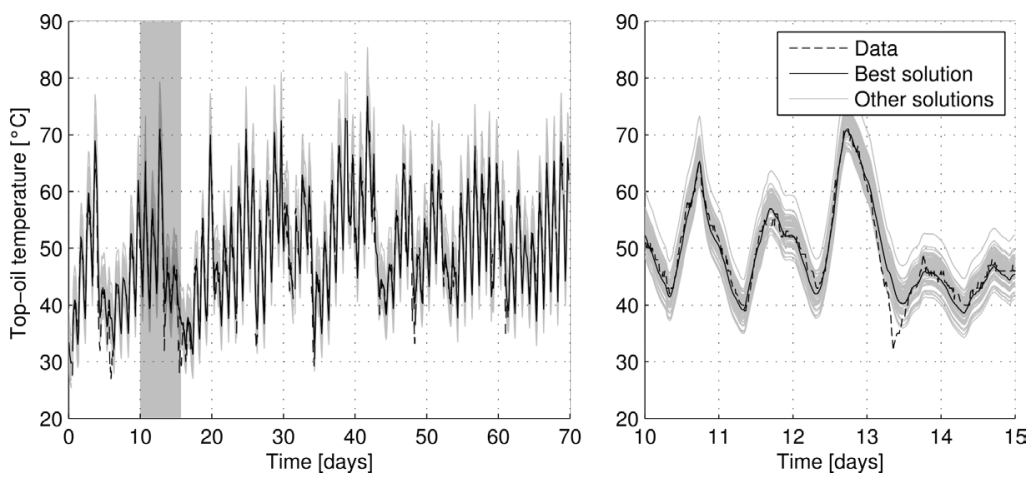

(b)
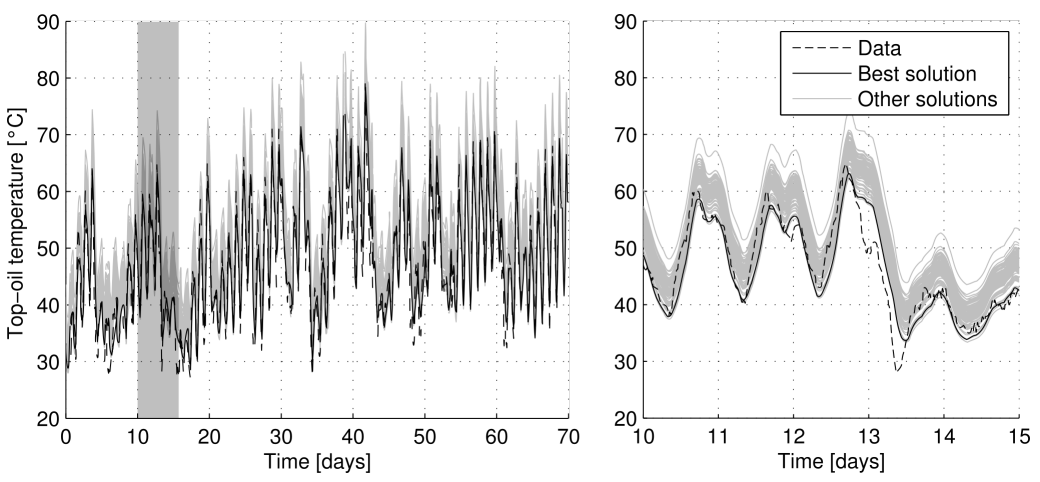

(c)

Figure 4. The graphical representation to visualize the variability of the solutions obtained with the Niching Genetic Algorithm and the corresponding top-oil temperature estimates. (a) Each solution is represented by a polygon line crossing the parallel axes (the black line is the best solution); (b) Temperature curves generated on the training set (the dotted line represent the real measurements and the black line represent the best estimation curve); (c) Temperature curves generated on the validation set. 
axis diagram, and the different temperature curves generated by the model when using each parameter combination. On the one hand, the parallel axis diagram in Figure 4(a) helps to easily visualize each solution, represented by a polygon line that traverses each axis, indicating the value for each adjusted parameter. It involved 100 individuals and 37 generations to obtain these solutions. The color of the lines represents the fitness value of each solution, where the darkest corresponds to the best fitness. On the other hand, Figure 4(b) shows the different temperature curves for the dataset used in the adjustment process (training set), which are very similar to each other. The same happens with the curves for the validation dataset that are shown in Figure 4(c). The black color line indicates the closest estimate curve to the real curve represented by the dotted line. The RMSE was used in order to obtain the best parameter set among all feasible solutions. It can be seen that the solution is not unique since several solutions allow top-oil temperature estimations with the same error level. The best parameter values are shown in Table 1, with the values proposed by the Loading Guides for easy comparison. The optimized values do not differ too much from the Loading Guides recommendations, except the oil time constant $\tau_{o}$. However, they are all within the expected bounds.

Table 3 shows the errors that resulted from using the parameters obtained with the optimization algorithm and the parameters recommended in the Loading Guides for determining easily the improvement achieved with the optimization. The error levels reached in the training and the validation dataset are shown for both cases. The RMSE and RMSE\% values are reduced by $60.6 \%$ in training and $63.7 \%$ in validation, indicating a general reduction of the magnitude of the error. An important reduction also occurs in the MBE values, being positive for the validation. The MBE sign indicates that there is a slight overestimation of the temperature in general. Regarding the correlation coefficient $R$, its value is higher using the optimized parameters, which indicates that the estimates have correctly captured the general tendency present in the measured data. This result is reflected in Figure 5, where the different oil temperature curves are shown, and it is clear that the model with optimized parameters generates a better-fitted curve than the model with Loading Guides parameters.

Regarding the adjustment of the parameter for the calculation of the relative aging rate, two historical measurements datasets were used. The first one

Table 3. Error values obtained at the top-oil temperature calculation for the two different transformer data sets using different parameter values (Loading Guides recommendation and optimized parameter values).

\begin{tabular}{cccccc}
\hline Parameters & Dataset & RMSE & RMSE\% & MBE & $R$ \\
\hline \multirow{2}{*}{ IEC Guide } & Training & 7.10 & 14.51 & 6.41 & 0.94 \\
& Validation & 11.66 & 25.34 & 10.67 & 0.89 \\
& Training & 2.80 & 5.72 & -1.33 & 0.96 \\
Optimized & Validation & 4.23 & 9.20 & 2.55 & 0.94 \\
\hline
\end{tabular}




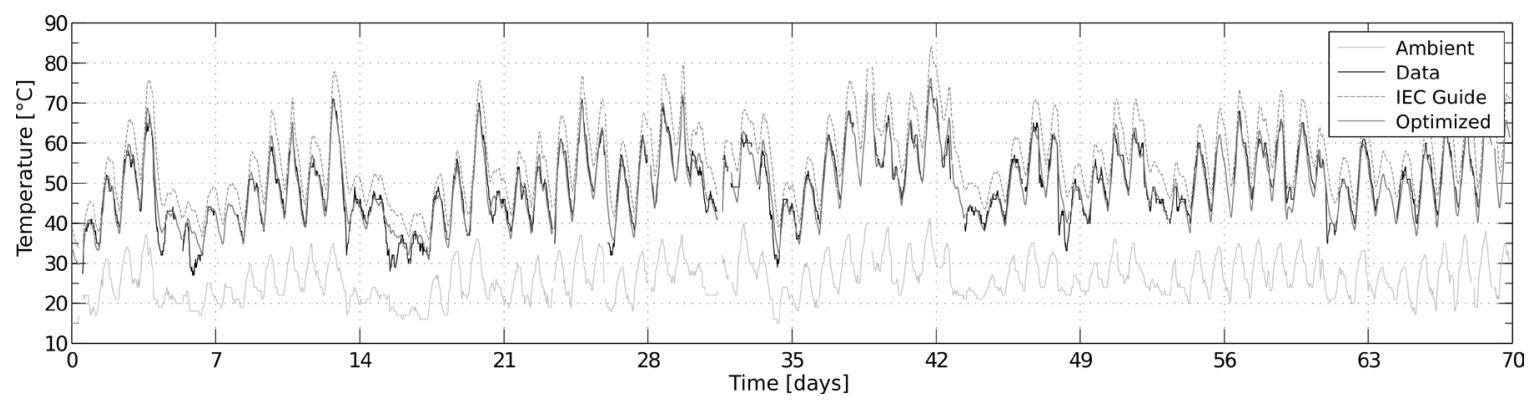

Figure 5. Comparison of the real measurements with the top-oil temperature estimates using parameters recommended by the IEC Loading Guide 2005 and the optimized parameters (the best solution obtained by GA). The ambient temperature is included for reference.

corresponds to a transformer installed and started working on 13-07-2001, and it was replaced by another transformer due to overload damage on 13-02-2016 (5328 operating days). The second case corresponds to a transformer put into operation on 02-08-2002, and damaged by overload on 23-03-2014 (4431 operating days). The measurements of these transformer substations are only available in the period between 01-01-2013 and 31-12-2016 (4 years of data). There is no record of movements or repairs conducted on any of the transformers, supported by old employees of the company that support the claim that they were never moved or repaired. The previous historical data was completed by replicating the available measurements in the missing periods, taking into account the annual consumption registered in the whole province, reported for residential customers. These annual increments were taken from statistical reports [23], and are shown in Figure 6(a) \& Figure 6(b) shows the current measurements for the first case (filled records are shown in gray color). A similar process was conducted for the second transformer. The exact date of the transformer critical overload damage is indicated by the dotted gray line. Finally, Figure 6(c) shows the accumulated loss of life, calculated in the entire time period analyzed. Then, the $B=11000$ is adopted in the Equation (1), for the calculated lifespan value to be equal to $100 \%$ at the time the transformers are damaged, For the calculated lifespan value to be $100 \%$ at the time the transformers are damaged, $B=11000$ is adopted in the Equation (7).

Finally, the thermal and the lifespan models adjusted in this paper are applied to the first case dataset to exemplify the use and implementation of a real time monitoring system. Figure 7 shows the resulting curves, highlighting the hottest period with the greater electricity demand. The measurements of the electrical consumption are shown in Figure 7(a) (the other two phases of the transformer are omitted for clarity); The temperatures in different levels of the transformer (hot-spot, top-oil and the ambient temperature) are shown in Figure 7(b); the relative aging rate curve is shown in Figure 7 (c), which indicates exactly when the lifespan reduction is greater; Finally, Figure 7(d) shows the evolution of accumulated loss of life, which allows us to determine how much useful life is left. By setting appropriate thresholds, an alarm system can be implemented based on 


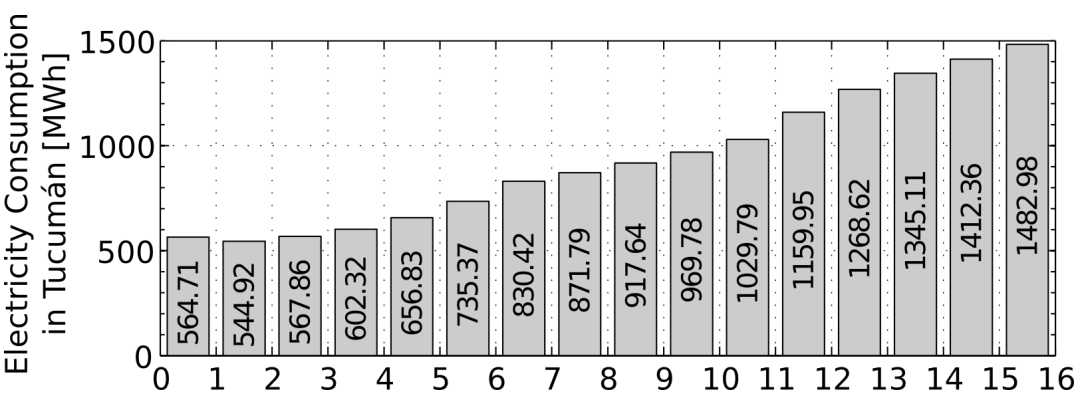

(a)

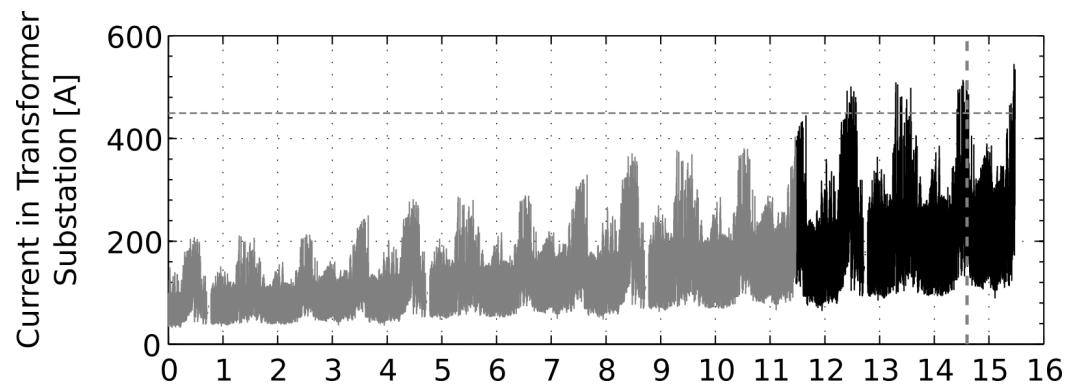

(b)

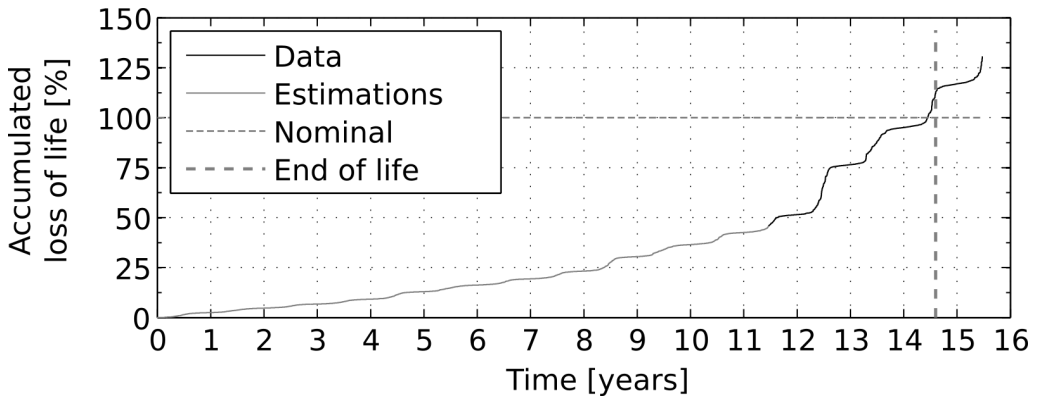

(c)

Figure 6. Data to fill the electrical consumption dataset and to adjust the parameters involved in the lifespan calculation procedure; (a) Annual consumption increments on the province of Tucumán for residential customers; (b) Available (black) and filled (gray) current measurements (the dotted gray line indicate the rated current); (c) Accumulated loss of life calculated using the adjusted parameter (the dotted gray line indicate when the transformer was damaged).

these curves, which would help during the decision making process when the transformers are at their top demand in the year.

\section{Conclusions}

Regarding the parameter adjustment of the top-oil temperature model, the optimized values are very similar to the values proposed by the Loading Guides, except for oil time constant $\tau_{0}$, which is slightly lower than expected. This difference may be due to the lack of adequate heat dissipation in the transformers so that it heats faster than normal, or may be attributable to the influence of the climate on heat dissipation or the particular characteristics of the transformer 

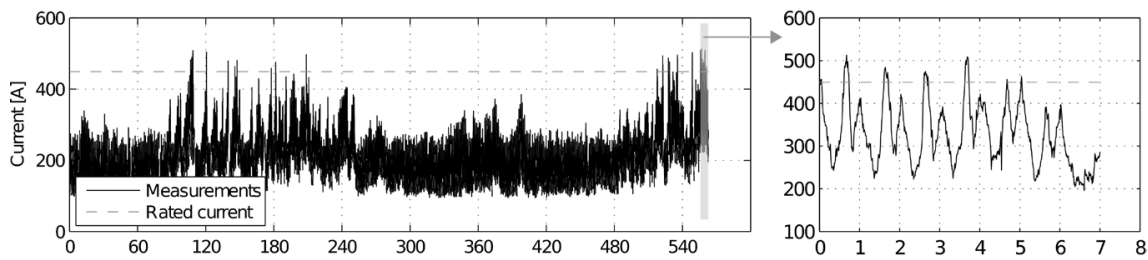

(a)
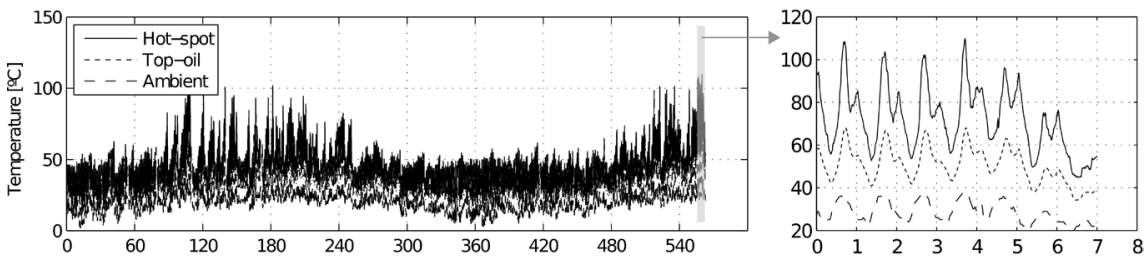

(b)
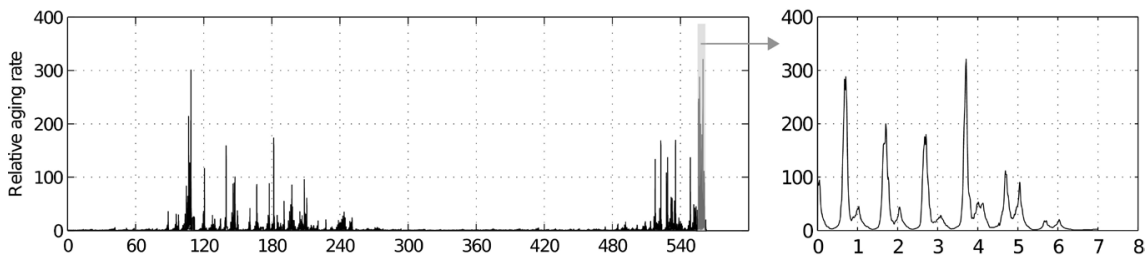

(c)
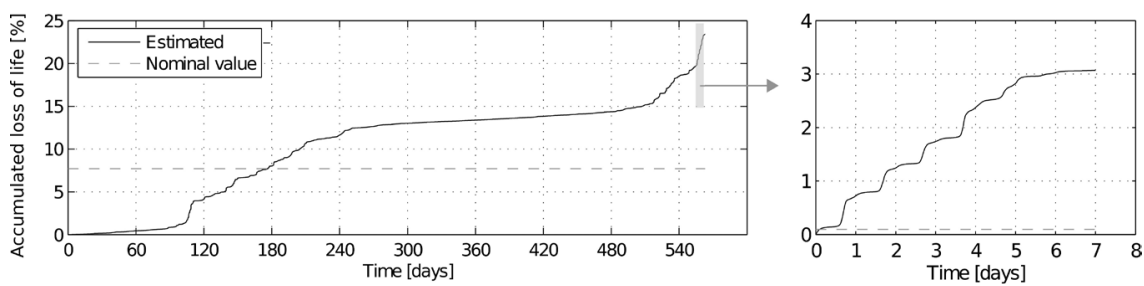

(d)

Figure 7. Visualization of some variables of interest in the monitoring of distribution transformers (on the right a short period is amplified). (a) Current measurements and current rate (dotted line); (b) The temperatures in different levels of the transformer; (c) the relative aging rate; (d) Accumulated and nominal loss of life.

used in the performed tests. These results indicate that the models proposed in the Loading Guides, once adjusted, are adequate for estimating the lifespan of the transformers located in the province of Tucumán. In this sense, the Genetic Algorithms allowed us to obtain good solutions. Some other restrictions might be added (e.g. based on the physics of the problem) that make the problem more complex, what might require adjustments or changes in the algorithm. If adding other restrictions to the parameter adjustment problem was necessary (possibly based on the physics of the problem), other algorithms could be used.

As for the adjustment of the aging rate equation, the results indicate that the lifespan is smaller than the values indicated in the Loading Guides. This result is a product of the particular insulation paper of these transformers degrades faster than expected due to ambient factors (high humidity, solar radiation influence, among others). However, we were unable to confirm this since, in this stage, the adjustment lacks enough data to produce solid results. To improve the accuracy 
of the prediction we need more data (transformers damaged by overload and with a traceable history). Nevertheless, the model and methodology presented in this paper constitute a good approximation that can be refined and improved as new data becomes available. The model adjustment and validation must be carried on continuously, due to the results are more precise when we have a greater number of measurements. It is important to notice that different brands and construction year seriously affect the transformer lifespan (transformers used to be built more solid and with a larger margin). So, even when more data become available, a clustering process should be performed in order to adjust a model to a group of transformers with similar strength.

From the results presented, we can conclude that the models and methodologies proposed allows for a correct implementation of a transformer monitoring system, helping to plan actions on the transformers near their end of life. As future work, we propose the inclusion of additional variables with greater impact on the internal temperature of the transformer, such as solar radiation [24] [25]. We will also explore the possibility of implementing Edge computing [26], where these calculation are performed right on the transformer and only an alarm is issued when necessary, is an interesting possibility that would greatly reduce communication issues.

\section{Acknowledgements}

This work was partially supported by grant UTN3870 for the research project "Intelligent Data Analysis Applied to Energy Management and Optimization". We also wish to extend thanks to Empresa de Distribución Eléctrica de Tucumán S.A. (E.D.E.T.s.a.) for providing the data necessary to elaborate this paper.

\section{Conflicts of Interest}

The authors declare no conflicts of interest regarding the publication of this paper.

\section{References}

[1] Fang, X., Misra, S., Xue, G. and Yang, D. (2012) Smart Grid-The New and Improved Power Grid: A Survey. IEEE Communications Surveys \& Tutorials, 14, 944-980. https://doi.org/10.1109/SURV.2011.101911.00087

[2] Government, U.S. (2011) NIST Framework and Roadmap for Smart Grid Interoperability Standards, Release 10 . General Books, Washington DC.

[3] Official Gazette of the Republic Argentina (2015) Energy Emergency, Decree 134/2015. Official Gazette of the Republic Argentina, Buenos Aires.

[4] Islam, M.M., Lee, G. and Hettiwatte, S.N. (2017) Application of a General Regression Neural Network for Health Index Calculation of Power Transformers. International Journal of Electrical Power \& Energy Systems, 93, 308-315. https://doi.org/10.1016/j.ijepes.2017.06.008

[5] Kazemi, R., Jazebi, S., Deswal, D. and de León, F. (2017) Estimation of Design Pa- 
rameters of Single-Phase Distribution Transformers from Terminal Measurements. IEEE Transactions on Power Delivery, 32, 2031-2039. https://doi.org/10.1109/TPWRD.2016.2621753

[6] Beltle, M. and Tenbohlen, S. (2016) Power Transformer Diagnosis Based on Mechanical Oscillations Due to ac and dc Currents. IEEE Transactions on Dielectrics and Electrical Insulation, 23, 1515-1522. https://doi.org/10.1109/TDEI.2016.005537

[7] Tenbohlen, S., Coenen, S., Djamali, M., Müller, A., Samimi, M.H. and Siegel, M. (2016) Diagnostic Measurements for Power Transformers. Energies, 9, 347. https://doi.org/10.3390/en9050347

[8] Hashmi, M., Hänninen, S. and Mäki, K. (2011) Survey of Smart Grid Concepts, Architectures, and Technological Demonstrations Worldwide. 2011 IEEE PES Conference on Innovative Smart Grid Technologies (ISGT LA), Medellin, 19-21 October 2011, 1-7.

[9] Commission, I.E. (2005) Loading Guide for Oil-Immersed Power Transformers (IEC 60076-7). IEC, London.

[10] IEEE (2012) IEEE Guide for Loading Mineral-Oil-Immersed Transformers and Step-Voltage Regulators. IEEE Std C57.91-2011 (Revision of IEEE Std C57.91-1995), $1-123$.

[11] Emsley, A.M. and Stevens, G.C. (1994) Review of Chemical Indicators of Degradation of Cellulosic Electrical Paper Insulation in Oil-Filled Transformers. IEE Proceedings-Science, Measurement and Technology, 141, 324-334. https://doi.org/10.1049/ip-smt:19949957

[12] Agarwal, V., Lybeck, N.J., Pham, B.T., Bickford, R. and Rusaw, R. (2015) Implementation of Remaining Useful Lifetime Transformer Models in the Fleet-Wide Prognostic and Health Management Suite. Proceedings of 9 th International Conference on Nuclear Plant Instrumentation, Control, \& Human-Machine Interface Technologies (NPIC-HMIT), Charlotte, 23-26 February 2015, 23-26.

[13] IEEE (1995) IEEE Guide for Loading Liquid-Immersed Step-Voltage and Induction-Voltage Regulators. ANSI/IEEE Std C57.95-1984. 0_1.

[14] Naguil, J.L., Szewczuk, O., Bahamode, P.J., Argañaraz, R., Orue, J.L., Calafiore, C.A., et al. (2011) Redes Eléctricas Inteligentes en Argentina. Redes Eléctricas Inteligentes En Argentina, Mar del Plata.

[15] Tripaldi, J.C. and Moreno, D.A. (2015) AEA 92559-1: Towards an Argentinean Smart Grid Vision. 2015 IEEE PES Innovative Smart Grid Technologies Latin America (ISGT LATAM), Montevideo, 5-7 October 2015, 768-773. https://doi.org/10.1109/ISGT-LA.2015.7381252

[16] Aster, R.C., Borchers, B. and Thurber, C.H. (2011) Parameter Estimation and Inverse Problems. Academic Press, Cambridge.

[17] Tang, W.H. and Wu, Q.H. (2011) Thermal Model Parameter Identification and Verification Using Genetic Algorithm. In: Condition Monitoring and Assessment of Power Transformers Using Computational Intelligence, Springer, London, 73-94. https://doi.org/10.1007/978-0-85729-052-6_5

[18] Galdi, V., Ippolito, L., Piccolo, A. and Vaccaro, A. (2001) Parameter Identification of Power Transformers Thermal Model via Genetic Algorithms. Electric Power Systems Research, 60, 107-113. https://doi.org/10.1016/S0378-7796(01)00173-0

[19] Eaton, J.W., Bateman, D., Hauberg, S. and Wehbring, R. (2015) GNU Octave Version 4.0.0 Manual: A High-Level Interactive Language for Numerical Computations. 
[20] Yu, X. and Gen, M. (2010) Introduction to Evolutionary Algorithms. Springer, Berlin. https://doi.org/10.1007/978-1-84996-129-5

[21] Holland, J.H. (1992) Adaptation in Natural and Artificial Systems: An Introductory Analysis with Applications to Biology, Control and Artificial Intelligence. MIT Press, Cambridge.

[22] Dick, G. (2005) A Comparison of Localised and Global Niching Methods. The 17 th Annual Colloquium of the Spatial Information Research Centre University of Otago, Dunedin, 24-25 November 2005, 91-101.

[23] Statistics Directorate of Tucumán Province (2016) Energy and Fuel Demand in Tucumán.

[24] Hajidavalloo, E. and Mohamadianfard, M. (2010) Effect of Sun Radiation on the Thermal Behavior of Distribution Transformer. Applied Thermal Engineering, 30, 1133-1139. https://doi.org/10.1016/j.applthermaleng.2010.01.028

[25] Gorgan, B., Notingher, P.V., Wetzer, J.M., Verhaart, H.F.A., Wouters, P.A.A.F. and Van Schijndel, A. (2012) Influence of Solar Irradiation on Power Transformer Thermal Balance. IEEE Transactions on Dielectrics and Electrical Insulation, 19, 1843-1850. https://doi.org/10.1109/TDEI.2012.6396939

[26] Shi, W., Cao, J., Zhang, Q., Li, Y. and Xu, L. (2016) Edge Computing: Vision and Challenges. IEEE Internet of Things Journal, 3, 637-646.

https://doi.org/10.1109/JIOT.2016.2579198 